\title{
Projeto Terapêutico Singular como Abordagem Multiprofissional no Hospital
}

The Single Therapeutic Project team Approach as in Hospital

Proyecto Singular Terapéutica como Enfoque Multidisciplinario en el Hospital

Camila Vasconcelos Carnaúba Lima ${ }^{1}$

Mairla dos Santos Rodrigues Moura ${ }^{2}$

Márcia Valéria da Silva Cavalcante ${ }^{3}$

\section{Resumo}

Objetivo: descrever a abordagem multiprofissional por meio do desenvolvimento do Projeto Terapêutico Singular num contexto hospitalar. Método: Foi apresentado um relato de experiência de profissionais residentes de diversas áreas sobre $\mathrm{o}$ processo de construção do Projeto Terapêutico Singular. A análise da experiência foi fundamentada nos conceitos de Wenger e Schön, sobre comunidades de prática e ação reflexiva, respectivamente, bem como na discussão dos temas levantados sobre clínica ampliada e abordagem multiprofissional. Resultados: O Projeto Terapêutico Singular pode favorecer a interação das áreas e a negociação de novos significados na atuação profissional. Trata-se de um possível instrumento de organização do trabalho visando à assistência integral do sujeito e à modelação de uma ética multiprofissional na medida em que sua prática é refletida e revisada. No entanto, ainda são muitos os desafios. Faz-se necessário ampliar as possibilidades de interação com outros profissionais não residentes na construção do Projeto

${ }^{1}$ Psicóloga. Discente do Programa de Mestrado em Psicologia Universidade Federal de Alagoas (UFAL). Autor correspondente: Av. Francisco Amorim Leão, 535, Pinheiro. CEP: 57057-780. Maceió - AL, Brasil. E-mail: camilacarnauba@gmail.com

${ }^{2}$ Farmacêutica. Pós-graduação pelo Programa de Residência Integrada Multiprofissional em Saúde do Adulto e do Idoso da UFAL

${ }^{3}$ Assistente Social. Pós-graduação pelo Programa de Residência Integrada Multiprofissional em Saúde do Adulto e do Idoso da UFAL.

Recebido: Mar/2016 - Aceito: Jun/2017. 
Terapêutico Singular, garantir a articulação com a rede de assistência e o acompanhamento do usuário após a alta hospitalar. Conclusão: $\mathrm{O}$ Projeto Terapêutico Singular pode fortalecer a abordagem multiprofissional, possibilitando humanizar as práticas em saúde e tendo como base a figura do usuário sob uma dimensão biopsicossocial. Espera-se que esse relato contribua com o trabalho de outras equipes de saúde e fomente debates, discussões e aprimoramentos das políticas de humanização.

\section{Descritores: Saúde; Hospital; Equipe.}

\section{Abstract}

Objective: to describe the multiprofessional approach through the development of the Unique Therapeutic Project in a hospital context. Method: It was presented an experience report of resident professionals from different areas on the construction process of the Unique Therapeutic Project. The analysis of the experience was based on the concepts of Wenger and Schön, on communities of practice and reflexive action, respectively, as well as on the discussion of the topics raised on expanded clinical and multiprofessional approach. Results:
The Unique Therapeutic Project can favor the interaction of the areas and the negotiation of new meanings in the professional performance. It is a possible instrument of work organization aiming at the integral assistance of the subject and the modeling of a multiprofessional ethics to the extent that its practice is reflected and revised. However, there are still many challenges. It is necessary to expand the possibilities of interaction with other non-resident professionals in the construction of the Unique Therapeutic Project, to ensure articulation with the care network and follow-up of the patient after discharge. Conclusion: The Unique Therapeutic Project can strengthen the multiprofessional approach, making it possible to humanize health practices and based on the figure of the user under a biopsychosocial dimension. It is hoped that this report contributes to the work of other health teams and encourages debates, discussions and improvements in humanization policies.

\section{Descriptors: Cheers; Hospital; Team.}

\section{Resumen}

Objetivo: Describir el enfoque multidisciplinario a través del 
desarrollo del Proyecto Singular terapéutico en un hospital. Método: A los residentes de la experiencia profesional de informes de diferentes áreas del proceso de construcción del Proyecto Singular terapéutico se presentó. El análisis del experimento se basa en los conceptos de Wenger y Schön en las comunidades de práctica y la acción reflexiva, respectivamente, y en la discusión de las cuestiones planteadas sobre el enfoque multidisciplinario ampliada y clínica. Resultados: Terapéutica de Proyecto Singular pueden favorecer la interacción de las áreas y la negociación de nuevos significados en el desempeño profesional. Esta es una posible herramienta de organización del trabajo dirigido a la atención integral del sujeto y el modelado de un multi-ética en la medida en que su práctica se refleja y revisado. Sin embargo, todavia hay muchos desafios. Es necesario ampliar las posibilidades de interacción con otros profesionales no residentes en la construcción del Proyecto Singular terapéutica, asegurar la coordinación con la red de servicios y el seguimiento del usuario después de la descarga. Conclusión: Terapéutica de Proyecto Singular puede fortalecer el enfoque multidisciplinario, lo que permite humanizar las prácticas de salud y basada en la figura del usuario en una dimensión biopsicosocial. Se espera que este informe contribuya a la labor de otros equipos de salud y promover debates, discusiones $y$ mejoras de las políticas de humanización.

Descriptores: Salud; Hospital; Equipo.

\section{Introdução}

A prática humanizada tem sido um desafio recorrente entre os trabalhadores do Sistema Único de Saúde, como também a visão sobre o usuário a partir da sua dimensão biopsicossocial. A Política Nacional de Humanização do Sistema Único de Saúde (HumanizaSUS) tem como estratégia aprimorar a produção de saúde e de assistência reorganizando as relações humanas, em que usuários, profissionais e gestores sejam corresponsáveis pelo planejamento, condução dos serviços, avaliação e administração dos conflitos advindos desta nova constituição de grupalidade A partir de então, recomenda-se o uso do Projeto Terapêutico Singular como um dispositivo que auxilia a transição da clínica tradicional médicobiologicista para a clínica ampliada humanizada, articulando a tríade 
equipe-usuário-família bem como construindo estratégias que visam à produção do cuidado e a assistência integral ao usuário ${ }^{(1)}$.

Trata-se de uma reunião da equipe multiprofissional para traçar propostas de ações biopsicossociais e deve ser desenvolvido em quatro momentos: 1) Diagnóstico biopsicossocial; 2) Definição de metas; 3) Divisão de responsabilidades; 4) Reavaliação do Projeto $^{(4)}$. Além disso, é essencial articular as diversas especialidades profissionais, caracterizadas pelos distintos saberes e práticas, distintas formas de visão sobre a problemática, de modo a organizar proposições comuns que expliquem à problemática, permitindo à equipe a composição de estratégias de intervenção que vão garantir maior sucesso e menores riscos de $\operatorname{agravo}^{(7)}$.

Considerando que a equipe de saúde é a principal via de organização dos serviços em saúde, o trabalho da equipe multiprofissional é fundamentado enquanto uma modalidade de trabalho coletivo que se caracteriza por múltiplas atuações técnicas e interação de diversas áreas profissionais cuja relação é recíproca ${ }^{(8)}$. E, ainda, requer flexibilidade da divisão de trabalho e autonomia técnica com interdependência. Por fim, a equipe multiprofissional integrada visa ao compartilhamento de uma ética, para além das técnicas ${ }^{(9)}$.

Dois conceitos principais dão suporte teórico ao presente artigo, a comunidade de prática e trabalho reflexivo. O primeiro é referenciado por Etienne Wenger ${ }^{(2)}$ e, o segundo, por Schön $^{(3)}$. Ambos abordam e elaboram princípios de aprendizagem em grupo. Wenger $^{(2)}$ define comunidades de práticas como grupos de pessoas que comungam interesses/atividades e encontram, por meio da interação entre elas, um meio mais eficiente de aprimorar suas ações. Dessa forma, existem aí preocupações ou paixões partilhadas por meio da interação regular de um determinado grupo, o qual seu desempenho remete ao aprimoramento de prática sobre aquilo que lhes são comuns.

Para Schön ${ }^{(3)}$ o profissional que é reflexivo tem uma visão de totalidade acerca do problema estudado, pois diferentemente do profissional especialista que olha o problema em seu recorte, o profissional reflexivo olha para a coisa em si e busca compreendêla em sua totalidade. Para o autor, o que proporciona a produção de pensamento reflexivo através da prática cotidiana é a aliança entre experiências profissionais e conhecimento científico. Nesse 
Lima CVC, Moura MSR, Cavalcante MVS 476

Projeto Terapêutico Singular como Abordagem Multiprofissional no Hospital

ínterim, é indispensável o planejamento de práticas e as reflexões coletivas de forma a produzir discussões nas quais haja a interação com o outro.

Assim, o presente artigo descreve considerações/impressões sobre a vivência do trabalho em equipe multiprofissional a partir do desenvolvimento do Projeto Terapêutico Singular por profissionais residentes de diversas áreas em atuação num contexto hospitalar.

\section{Método}

Esse estudo apresenta um relato de experiência de profissionais residentes na implantação do Projeto Terapêutico Singular na Clínica Médica de um hospital universitário localizado na capital alagoana. Diversas áreas se envolveram na construção desse Projeto como Enfermagem, Nutrição, Psicologia, Farmácia e Serviço Social.

Esse relato buscou dialogar com os conceitos que norteiam o trabalho em equipe propostos por Wenger ${ }^{(2)} \mathrm{e}$ Schön ${ }^{(3)}$ - as comunidades de prática e a ação reflexiva, respectivamente. Ademais, também se articulou com discussões de outros temas relevantes como clínica ampliada e abordagem multiprofissional - e levantou algumas reflexões acerca da prática exposta.

\section{Resultados e Discussão}

Iniciamos o processo de construção do Projeto Terapêutico Singular com a seleção de um paciente preferencialmente recém-admitido no hospital e com maior vulnerabilidade biopsicossocial. Diante disso, realizamos uma abordagem multiprofissional onde o acolhemos e ofertamos uma escuta qualitativa. Buscamos, então, efetivar as quatro etapas propostas pelo Projeto Terapêutico Singular, traçando um diagnóstico, definindo metas, dividindo responsabilidades e reavaliando ações e resultados. O usuário hospitalizado e seus familiares também são inteirados sobre o planejamento da equipe com intuito de promover condições de coelaboração destes nesse processo. Tais práticas condizem com as orientações da Política Nacional HumanizaSUS ${ }^{(4)}$.

$\mathrm{Na}$ atuação multiprofissional, dividimos responsabilidades para execução do Projeto, no entanto, buscamos compartilhar os mesmos objetivos de modo que nossos trabalhos estejam sincronizados. Debatemos os casos de maneira interdisciplinar, embora algumas profissões exerçam um protagonismo maior quando comparado à outra em determinados momentos. 
Nesse momento, somos desafiados a interagir entre diferentes áreas. E, para que isso se tornasse possível, foi indispensável a inclusão da atividade de elaboração do Projeto Terapêutico Singular na carga horária do residente. O horário fixo programado para as reuniões semanais facilita a execução das atividades discursivas/dialógicas e de planejamento da equipe. Do mesmo modo, as reuniões informais - dos corredores ao refeitório $-\mathrm{e}$ aquelas realizadas em tempos imprevistos também são válidas nesse processo.

Wenger $^{(2)}$ considera que a informalidade é uma característica, não menos importante, da comunidade de prática. Segundo o autor, os membros de uma comunidade de prática possuem vínculos informais, o que promove a comunicação e, por conseguinte, esta pode ser resolutiva e reflexiva até nos encontros não formais. Schön ${ }^{(3)}$ implementa esse aspecto no trabalho crítico e reflexivo. De acordo com o autor, é no dia a dia que as hipóteses teóricas definidas pelo grupo devem ser checadas. Isso permite que discussões e avaliações sobre a prática profissional ocorram com frequência durante interação cotidiana dos membros do grupo.
Nossa busca pela comunicação qualitativa ao discutir sobre os casos selecionados repercutiu no desenvolvimento de uma escuta mais acolhedora e até terapêutica no atendimento ao usuário. A interação de nossas áreas permitiu a integração dos vários olhares profissionais admitindolhe em suas diversas possibilidades de existência e de necessidades. Procuramos constituir um vínculo equipe-usuário, possibilitando conhecer a singularidade do sujeito de maneira contínua.

Faz-se necessário compreender as peculiaridades da história de vida do usuário hospitalizado expressas pelos seus desejos e dinâmica sociofamiliar ${ }^{(6)}$. Isso traduz a importância da clínica ampliada compreendida como um compromisso firmado com o sujeito e o seu coletivo, tratando o problema de saúde a partir da sua dimensão biopsicossocial $^{(5)}$.

Entende-se que a percepção da singularidade foi possível a partir do trabalho em equipe que, conforme Oliveira $^{(7)}$, congrega os diversos olhares sobre a problemática, saberes e práticas. Tal prática permite o compartilhamento de ideias entre os membros da equipe e os auxilia no processo de composição de estratégias interventivas sobre determinada problemática. Assim, é 
possível diminuir os riscos de agravo na saúde dos usuários. As distintas formações profissionais possibilitaram a visão de totalidade, característica do profissional reflexivo, como pontua Schön ${ }^{(3)}$.

Nessa perspectiva, a utilização do Projeto Terapêutico Singular - como base para a abordagem multiprofissional - proporcionou a interação das diferentes áreas, considerando que foi possível organizar a prática multidisciplinar ao tempo que atendia as necessidades do usuário e seus familiares. Conforme Oliveira $^{(7)}$, o Projeto Terapêutico Singular trata-se de um dispositivo de intercâmbio entre equipe multiprofissional e usuários, em que ambos se beneficiam, pois permite a articulação e organização de equipes de profissionais e, aos usuários, possibilitam a restauração de suas relações afetuosas e sociais, como também a reconquista de direitos e poder social. Ou seja, o atendimento das necessidades objetivas e subjetivas do usuário.

Nesse processo de desenvolvimento do Projeto Terapêutico Singular, a interação de áreas é permeada pelo exercício do diálogo, considerando a negociação das diferentes opiniões profissionais que buscavam a solução do problema e a melhor assistência ao usuário. Assim, segundo Pereira e Harris ${ }^{(8)}$, a equipe exercita a "multiprofissionalidade", onde cada profissional contribui com seu conhecimento na resolução da problemática, podendo assim gerar resultados satisfatórios.

A interação de áreas trata-se de uma característica indispensável presente no trabalho multiprofissional. Wenger $^{(2)}$ caracteriza a comunidade de prática, seu principal conceito, pela participação de pessoas dispostas a criar, refinar, comunicar-se e usar conhecimento para fazer diferença em sua prática. É, dessa forma, uma dinâmica de aprendizagem diferenciada e que se inicia através da interação.

Contudo, ainda nos deparamos com muitos desafios e dificuldades no quesito de interação profissional. Um deles implica em envolver outros profissionais do setor (por exemplo, médicos, enfermeiras, técnicos de enfermagem) nas fases de construção do Projeto Terapêutico Singular. Ainda são insuficientes nossas condições de interação com esses profissionais (residentes médicos ou funcionários) que atendem os mesmos pacientes durante sua hospitalização. Além disso, também encontramos dificuldades de manter contato com a rede de assistência, o que reduziu as 
possibilidades de co-responsabilização de outras instituições na continuidade do trabalho multiprofissional. Por fim, outra dificuldade encontrada se refere a nossa falta de participação na decisão da alta hospitalar, o que nos impedia de "finalizar" a etapa de hospitalização do usuário com os devidos desfechos visando à continuidade do cuidado.

No que se trata das dificuldades encontradas, faz-se necessário, ampliar a interação em redes qualitativamente. Outras unidades de saúde não só precisam ser mais acionadas, como também mais co-participativas na continuidade, efetividade e até de construção do Projeto Terapêutico Singular.

Por outro lado, a interação de nossas áreas também deu ensejo à renegociação de novos significados a cada profissão. Frequentemente colocávamos à prova a exclusividade de protocolos específicos. $\mathrm{Na}$ verdade, incrementávamos novos valores às nossas visões de ofício no manejo de atendimentos aos usuários. $\mathrm{Na}$ construção do planejamento estratégico fundamentado no Projeto Terapêutico Singular, a consequência das discussões promovidas por ele inferiu no compartilhamento de objetivos entre nossas atuações.
À medida em que discutíamos e revisávamos constantemente nosso modo de atuação perante os casos trabalhados, reflexões sobre nossa própria prática profissional vinham à tona. Dessa forma, foi difícil limitar o objetivo de um atendimento especializado ao interesse, apenas, de uma determinada área. Nossos objetivos, enquanto equipe multiprofissional, não somente eram visados/cumpridos num único atendimento, mas de modo contínuo perpassando as discussões e os diversos atendimentos durante todo período de hospitalização do usuário. Ademais, a interação da equipe que promovia a intersecção de objetivos foi além de definição de metas. Por trás da sistematização do Projeto Terapêutico Singular, criava-se uma oportunidade de compartilhar uma ética, conforme citado por Peduzzi ${ }^{(9)}$, negociando novos significados. Nesse contexto, o usuário, participante do Projeto Terapêutico Singular, se beneficiava com a continuidade e integralidade das ações da equipe.

Essa característica ratifica o que se define enquanto "comunidade de prática", segundo Wenger ${ }^{(2)}$. Espera-se ir além de um conjunto de relações num mesmo espaço geográfico, mas de construção de identidade à medida que a 
interação entre os membros molda seus modos de aprender/conhecer/pertencer. Tal modelação produz um sentimento de pertencimento da equipe já que compartilham valores, não somente tarefas e agendas.

Outro aspecto a se considerar diz respeito à autonomia dos usuários atendidos durante sua vivência hospitalar. Campos e Amaral ${ }^{(1)}$ apontam que a autonomia do sujeito pode ser avaliada pela sua capacidade de compreensão e atuação sobre o seu meio. Nas reuniões do Projeto Terapêutico Singular frequentemente era levantada a questão da autonomia do usuário em acessar informações sobre sua real condição clínica. Entendemos que autonomia é o poder do outro decidir sobre sua própria vida, de autogovernar-se. Percebemos, então, que não se trata apenas do direito de saber sobre seu problema físico de saúde, mas de instrumentalizar o usuário para decidir quando saber, como saber, e de instrumentalizar a família para preparar o mesmo para enfrentar a problemática. O que podemos observar é que, por meio da abordagem multiprofissional, a reflexão da ação e a mediação equipe-usuário-médico, favoreceu ao usuário a fortalecer seu poder de questionar sobre sua problemática de saúde, sobre os resultados dos exames, a decidir sobre as possibilidades terapêuticas assim como seu poder de decisão sobre a condução do contexto sócio-familiar. Dessa forma, os novos significados não são somente negociados entre nós, residentes profissionais, mas também pelo próprio usuário que também acompanha e constrói o Projeto Terapêutico Singular.

Todavia, ainda existem desafios e limitações nesse processo. Há muito a ser compartilhado com outros profissionais. O pouco envolvimento da equipe de residentes com outros profissionais do setor restringe $\mathrm{o}$ conhecimento apenas àqueles que participavam do Programa de Residência em foco. Foi possível promover interação de nossas áreas e até negociação de novos significados de prática profissional, mas também poderia agregar toda a equipe do setor em que o usuário esteve hospitalizado. Essa é uma proposta em discussão na equipe multiprofissional que poderia ser efetivada nas próximas oportunidades.

\section{Conclusão}

O objetivo desse artigo foi relatar a experiência de residentes de várias áreas profissionais sobre o trabalho 
multiprofissional numa instituição hospitalar. A equipe buscou orientar-se pela construção do Projeto Terapêutico Singular, um meio de abordagem multiprofissional sustentado por princípios da clínica ampliada. Criar ambientes favoráveis à interação das áreas - horários e locais fixos de reuniões, estudos, discussões e redação do planejamento - foi o primeiro passo para esse trabalho. Contudo, à medida que o projeto se desenvolvia, a equipe precisou ser flexível para as possibilidades de interagir em outros cenários, aqueles encontros informais em horários imprevisíveis no cotidiano do hospital (ou não).

Todavia, para além do cumprimento das etapas propostas pelo Projeto Terapêutico Singular, existiram outros aprendizados no que se trata de novos modos de compreender e, por conseguinte, se relacionar com o usuário. A equipe, a todo instante precisou avaliar-se, além de renegociar seus próprios valores pessoais/profissionais em troca do compartilhamento de uma ética e identidade focada na assistência integral do usuário.

As condições de interação das áreas e de renegociação de novos significados na atuação profissional, vai ao encontro das bases de trabalho em equipe referenciadas por Wenger ${ }^{(2)}$ e Schön ${ }^{(3)}$. A busca por auto-organização, planejamento e avaliação das ações da equipe iniciou o processo de criação de comunidade de prática bem como o desenvolvimento de novas habilidades profissionais por meio da ação reflexiva.

Ainda são muitos os desafios, mas a experiência de ampliar as possibilidades profissionais para $\mathrm{o}$ trabalho coletivo gerou resultados e sentimentos benéficos e resolutivos para todos os membros envolvidos. Espera-se que esse relato contribua com o trabalho de outros profissionais e equipes de saúde, dando-lhes suporte, estimulando reflexões e expressando as dificuldades do percurso para aqueles que também desafiam a capacidade do trabalho multiprofissional. Além disso, que ele fomente debates, discussões e aprimoramentos das políticas de humanização. Inclusive, há de se questionar se a realidade brasileira dos serviços de saúde tem utilizado o Projeto Terapêutico Singular enquanto estratégia de cuidado integral aos usuários hospitalizados.

\section{Referências}


1. Campos GWS, Amaral MA. A clínica ampliada e compartilhada, a gestão democrática e redes de atenção como referenciais teórico-operacionais para a reforma do hospital. Saúde Coletiva (Campinas) [periódico na internet]. 2007 [citado 2015 fev. 27]; 12(4): 849-59. Disponível em: http://www.scielo.br/pdf/csc/v12n4/04.p df

2. Wenger E. Communities of practice: a brief introduction; 1998. Disponível em: http://wenger-trayner.com/theory/.

3. Schön D. The Reflective Practioner: how professional think in action. New York: Basic Books; 1983.

4. Ministério da Saúde (BR). Clínica ampliada, equipe de referência e projeto terapêutico singular. Brasília: Ministério da Saúde; 2008.

5.Ministério da Saúde (BR). HumanizaSUS: Clínica ampliada. Brasília: Ministério da Saúde; 2004.

6. Barros JO. A construção de projetos terapêuticos no campo da saúde mental: apontamentos acerca das novas tecnologias de cuidado. São Paulo. Tese [Mestrado em ciências] - Faculdade de Medicina da Universidade de São Paulo; 2010. In: Biblioteca digital USP. Disponível em: http://www.teses.usp.br /teses/disponiveis/5/5163/tde-21092010 -093913/pt-br.php .

7. Oliveira GN. O projeto terapêutico singular. In: Campos GWS, Guerrero AVP, organizadores. Manual de práticas em Atenção Básica: saúde ampliada e compartilhada. Rio de Janeiro: Hucitec; 2007; 283-97.
8. Pereira IMTB, Harris WM. Estágio integrado na faculdade de saúde pública da Universidade de São Paulo: preparo para o trabalho multiprofissional. Saúde Pública (São Paulo) [periódico na Internet]. 1976 [citado 2015 fev. 27]; 10: 257-66. Disponível em: http://www.scielo.br/pdf/rsp/v10n3/06.p df

9. Peduzzi M. Equipe multiprofissional de saúde: conceito e tipologia. Saúde Pública (São Paulo) [periódico na Internet]. 2001 [citado 2015 fev. 27]; 35:103-09. Disponível em: http://www. scielo.br/scielo.php?script $=$ sci_arttext\& pid=S0034-89102001000100016. 